\title{
Does the metaphysical dog wag its formal tail? The free energy principle and philosophical debates about life, mind, and matter
}

\author{
Wanja Wiese (wanja.wiese@rub.de) \\ Institute of Philosophy II, Ruhr University Bochum, Germany
}

This paper is a commentary on Bruineberg, Dolega, Dewhurst, and Baltieri (forthcoming). The Emperor's New Markov Blankets. Behavioral and Brain Sciences. https://doi.org/10.1017/S0140525X21002351

\begin{abstract}
According to Bruineberg and colleagues, philosophical arguments on life, mind, and matter that are based on the free energy principle (FEP) (i) essentially draw on the Markov blanket construct and (ii) tend to assume that strong metaphysical claims can be justified on the basis of metaphysically innocuous formal assumptions provided by FEP. I argue against both (i) and (ii).

\section{Main text}

Bruineberg et al. distinguish between Markov blankets (MBs) as properties of models (epistemic 'Pearl blankets', PBs) and MBs as properties of physical systems (metaphysical 'Friston blankets', FBs). Unlike PBs, FBs are not exclusively defined in terms of probabilistic relations between random variables. Instead, FBs are also defined in terms of causal relations to a system's internal and external states: FBs comprise sensory and active states that mediate causal influences between internal and external states. In perceiving agents, this causal interplay between internal, external, and blanket states is supposed to capture perception-action loops (Friston, 2012).
\end{abstract}

As Bruineberg et al.'s lucid analysis shows, a realist interpretation of the notion of an FB is metaphysically demanding: positing the existence of an FB entails a statement about the physical system, i.e., it entails the existence of a boundary between internal and external states. Furthermore, the authors argue that the formalism that is used to identify internal, active, sensory, and external states (as in Friston, 2013) presupposes specific, non-arbitrary assumptions about the underlying model (target paper, sect. 4.1). This means that FBs are not theoretically neutral descriptions of a model's features. Furthermore, many descriptions of FBs in the literature are not metaphysically neutral, either, because they describe these blankets not just as properties of a model, but as boundaries of physical systems. As the authors point out, this is problematic to the extent that such metaphysical interpretations are assumed to "follow from the formal details" (target paper, sect. 4.2).

In the context of FEP, the notion of an MB is not just used to describe perception-action loops in certain self-organising systems. The distinction between internal, blanket, and external states is also invoked to describe the system's internal states as 
representations of probability distributions (of external states, given blanket states). According to FEP, this enables a dual description of the system's dynamics: on the one hand, in terms of the probabilistic evolution of internal states; on the other hand, in terms of the evolution of probabilistic beliefs about external states, parameterised by internal states (see Friston, 2019; Friston et al., 2020; see also Sprevak, 2020; Kiefer, 2020).

Apart from claims about the boundaries of minds, most philosophical claims about the relationships between life, mind, and matter do not essentially draw on the MB construct provided by FEP. Contrary to what Bruineberg et al. suggest, the main philosophically fruitful idea afforded by FEP is that internal states can be regarded as probability distributions over external states, given sensory and active states (see the examples given below).

One could object that this presupposes the existence of a boundary between a system and its environment-which, as the authors argue, cannot be derived from FEP, without presupposing strong metaphysical assumptions. We can grant this for the sake of argument, because the assumption that there is a boundary between a system and its environment is shared by many metaphysical theories of the mind (e.g., functionalism and, arguably, most versions of property physicalism), and none of these theories shows how to derive this partition from metaphysically innocuous assumptions. As long as FEP-based philosophical accounts avoid the mistake of assuming that FEP provides a metaphysically neutral way of determining boundaries, it is therefore not particularly problematic to posit the existence of a boundary between internal and external states (i.e., if it is problematic, it is problematic independently of FEP).

Bruineberg et al. argue that claims about the relationships between life, mind, and matter must make further metaphysical assumptions that do not follow from FEP. The authors worry that these assumptions "may in the end be doing all of the interesting work themselves" (target paper, sect. 7). I disagree with this statement, but here I will focus on the concern, expressed by the authors, that researchers mistakenly believe the FEP can be used to "settle fundamental metaphysical questions" (target paper, sect. 1).

In contrast to what Bruineberg et al. insinuate, the fact that additional assumptions are needed to contribute to metaphysical debates is acknowledged in FEP-based arguments for philosophical claims. Let me illustrate this with three examples (involving my own work).

In Friston et al. (2020), we argue that the most parsimonious interpretation of FEP's dual description of internal states is a form of property physicalism (reductive materialism) which we call Markovian monism. Crucially, we do not assume that this interpretation follows from the formalism itself: we consider and evaluate different metaphysical interpretations of the formalism (Friston et al., 2020, pp. 17-21).

Wiese \& Friston (2021a) argue that FEP is compatible with a strong continuity between life and mind. Again, this is not assumed to follow directly from the formalism. Rather, we explicitly point out that we presuppose a mechanistic account of physical computation and a representationalist interpretation of predictive processing / active inference (Wiese \& Friston, 2021a, pp. 10-13). 
In Wiese \& Friston (2021b), we draw on the duality between the probabilistic evolution of internal states and the evolution of probabilistic beliefs (entailed by FEP) to argue: a selforganising system is a conscious system (as opposed to a mere simulation of a conscious system) only if computational correlates of consciousness (CCCs) are instantiated by the physical processes that help the system sustain its existence (for details, see Wiese \& Friston, 2021b, pp. 22-24). FEP is here primarily invoked to provide a precise description of how a conscious system differs from a mere simulation: the dynamics of CCCs (in terms of probabilistic beliefs encoded by internal states) must be equivalent to the dynamics of internal states in terms of the system's non-equilibrium steady-state density.

In sum, I disagree with the authors' claim that FEP-based philosophical debates about the relationships between life, mind, and matter presuppose that FEP "can be used to settle fundamental metaphysical questions" (target article, sect. 1). Does this mean that the metaphysical heavy lifting is not done by FEP itself, but by additional metaphysical assumptions? I don't think so: philosophical arguments require clear concepts, and this is exactly what FEP's dual description of system dynamics affords.

\section{References}

Friston, K. (2012). A free energy principle for biological systems. Entropy, 14(11), 2100 2121. https://doi.org/10.3390/e14112100

Friston, K. (2013). Life as we know it. Journal of The Royal Society Interface, 10(86). https://doi.org/10.1098/rsif.2013.0475

Friston, K. (2019). A free energy principle for a particular physics. https://arxiv.org/abs/1906.10184

Friston, K. J., Wiese, W., \& Hobson, J. A. (2020). Sentience and the origins of consciousness: From Cartesian duality to Markovian monism. Entropy, 22(5), 516. https://doi.org/10.3390/e22050516

Kiefer, A. B. (2020). Psychophysical identity and free energy. Journal of The Royal Society Interface, 17(169), 20200370. https://doi.org/10.1098/rsif.2020.0370

Sprevak, M. (2020). Two kinds of information processing in cognition. Review of Philosophy and Psychology, 11(3), 591-611. https://doi.org/10.1007/s13164-019-004389

Wiese, W., \& Friston, K. J. (2021a). Examining the continuity between life and mind: Is there a continuity between autopoietic intentionality and representationality? Philosophies, 6(11), 18. https://doi.org/10.3390/philosophies6010018

Wiese, W., \& Friston, K. J. (2021b). The neural correlates of consciousness under the free energy principle: From computational correlates to computational explanation. Philosophy and the Mind Sciences, 2, 9. https://doi.org/10.33735/phimisci.2021.81 